\title{
Focal Plane Detector Array Developments
}

\author{
Zoran Ninkov, MEMBER SPIE \\ Rochester Institute of Technology \\ Imaging Science Center \\ 54 Lomb Memorial Drive \\ Rochester, New York 14623-5604
}

\author{
William J. Forrest \\ University of Rochester \\ 206 Bausch and Lomb Building \\ Physics and Astronomy Department \\ Rochester, New York 14627 \\ [DOI: $10.1117 / 1.1485760]$
}

The development of two-dimensional image sensors along with the development of affordable personal computing has greatly expanded the capabilities of image data acquisition and analysis. Image sensors today are used routinely in instrumentation ranging from consumer imaging devices to spaceborne telescopes. The most successful of such devices, to date, has been the charge coupled device (CCD). Its performance has seen continual improvement over the last two decades. Even so, there are still parameters, such as sub-pixel sensitivity variation, that need to be investigated. CMOS sensors provide the possibility of very good performance but at reduced cost and with capabilities not available on the CCD (true random addressibility and non-destructive readout). Most sensors need to be used behind a spectral filter (colored glass, interference filter, grating, etc.) to permit useful data to be extracted. This might for example be needed for color rendering of a scene or object detection in remote sensing or astronomy. Clearly integrating the functions of filtering and imaging would allow for more compact, flexible sensors. The ideal detector would offer the opportunity to not only record the location of photons on a focal plane but also record their energy. Such sensors could afford, for example, completely flexible spectral passbands. Thus a goal for imager scientists and engineers is the development of a true solidstate multispectral imager with large format, high sensitivity, low readnoise, room temperature operation, and reasonable energy resolution. This collection of papers presents current investigations in this continually developing field.

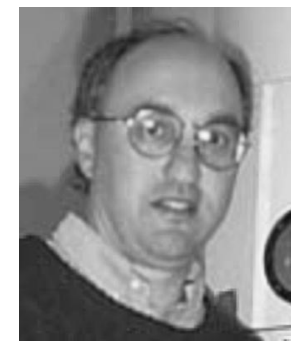

Zoran Ninkov completed his BSc (Honours) in physics at the University of Western Australia, his MSc in physical chemistry at Monash University, and his $\mathrm{PhD}$ in astronomy at the University of British Columbia in Vancouver, Canada. Subsequently, he has held positions in the Department of Physics and Astronomy at the University of Rochester, the Defense Science and Technology Organization in Australia, and the Institute for Space and Terrestrial Science in Toronto. He is currently a Professor of Imaging Science in the Center for Imaging Science at the Rochester Institute of Technology. His current research interests are in the development of new imaging arrays, testing and evaluation of arrays, and the development of new scientific instrumentation.

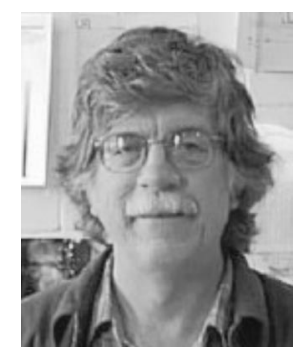

William Forrest received his BS in physics from Antioch College (1968) and his $\mathrm{PhD}$ in physics from the University of California, San Diego (1974). After research positions at the University of California, San Diego and Cornell University, he joined the University of Rochester, Department of Physics and Astronomy, where he is currently Professor of Astronomy and Director of the C. E. K. Mees Observatory. His primary research interest is in the area of infrared astronomy. In particular, he has focused on the nature of dust in and around stars and in the interstellar medium, star formation, and investigations of brown dwarfs. To this end, he is developing advanced infrared array detectors for cameras and spectrometers on ground-based and space observatories. 